\title{
Dolor pélvico crónico en la mujer
}

\author{
Pío Iván Gómez Sánchez*; Patricia Silvestre**; Mauricio Bonilla***; Consuelo Cayuela****; \\ Antonio Medina*****; Sara Fernández, Pilar Sánchez******
}

\begin{abstract}
RESUMEN: Se considera dolor pélvico crónico (DPC), aquél cuya duración no es inferior a seis meses y es uno de los motivos de consulta más frecuentes en Ginecología, no siendo fácil su enfoque diagnóstico ni terapéutico por la multiplicidad de causas que lo desencadenan, así como los factores psicógenos y socioculturales asociados.

Se revisan en este artículo, la neuroanatomía de los genitales internos femeninos, las etiologías más frecuentes de DPC y el enfoque diagnóstico, haciendo énfasis en la necesidad de equipos multidisciplinarios para su esłudio y manejo, siendo pilares diagnósticos la historia clínica detallada y la endoscopia ginecológica (cervicohisteroscopia-laparoscopia).
\end{abstract}

PALABRAS CLAVES: Dolor pélvico crónico, equipo multidisciplinario, endoscopia ginecológica.

SUMMARY: It is considered pelvic chronic pain that one wich duration is not inferior to six months, being one of the more frequent reasons for consultation in gynecology, neither its diagnosis nor its therapy are easy to determine, due to multiple causes that make it develop. Also the psycogenic, social and cultural factors that are associated with it.

In this article it is revised the neuroanatomy of the internal female sex organs, the more frequent etiology of the pelvic chronic pain and the diagnosis, making emphasis in the neccesity of multidisciplinary teams for its study and management, being the detailed clinic history an the gynecologic endoscopy its diagnostic pillars.

KEY WORDS: Pelvic chronic pain, multidisciplinary teams, gynecologic endoscopy.

\section{Introducción}

El dolor pélvico crónico (DPC), lo definimos, como aquél que tiene por lo menos seis meses de duración y es uno de los motivos de consulta más frecuentes en las clínicas ginecológicas. En el Instituto Materno Infantil de Santafé de Bogotá (IMI), ocupó el cuarto lugar como motivo de consulta en el área de consulta externa y fue la indicación de las dos terceras partes de las laparoscopias realizadas en los dos últimos años.

En Estados Unidos fue la causa del $12 \%$ de las histerectomías realizadas en un año (1).

Con el dolor agudo, estamos muy familiarizados, siendo en éste el diagnóstico más fácil y el tratamiento más efectivo, mientras que en el dolor crónico el diagnóstico diferencial es más difícil por la multiplicidad de etiologías, por la frecuente interferencia de factores psicológicos y por las diferencias socioculturales en la percepción del dolor, que obligan a crear equipos multidisciplinarios para el estudio y manejo

\footnotetext{
* Profesor Asistente. Dpto. Ginecobstetricia. Universidad Nacional de Colombia. Coordinador Clínica de Dolor IMI.

** Psicóloga Universidad Nacional. Clínica de Dolor IMI.

*** Psicólogo Universidad Nacional. Clínica de Dolor IMI.

**** Psicóloga Universidad Nacional. Clínica de Dolor IMI.

***** Anestesiólogo-Psiquiatra. Clínica de Dolor IMI.

****** Estudiantes Trabajo Sccial. Universidad Nacional. Clínica de Dolor IMI.
}

del DPC (2). A continuación se hará una revisión de la neuroanatomía, neurofisiología, características del DPC, así como un recuento de su etiología y el enfoque diagnóstico que se realiza en la Clínica de Dolor del IMI.

\section{Inervación de genitales femeninos}

Es importante hacer una revisión breve de las vías nerviosas, para entender mejor la localización e irradiación del dolor pélvico de origen ginecológico.

La inervación de la pared abdominal baja y parte anterior de la vulva está dada por los nervios ileohipogástrico, ileoinguinal y genitocrural. Impulsos del periné y parte baja de la vagina son llevados por ramas de nervios pudendos que vienen de segmentos S2, S3, S4.

La porción externa de las trompas y los ovarios están inervados por el plexo ovárico, cuyas fibras viajan junto a los plexos aórtico y renal hasta segmentos T10 T11 T12 L1; las cifras aferentes del ovario son principalmente componentes de T10 y las de la porción externa de la trompa T11 T12 L1. (Ver figura 1.)

Los estímulos dolorosos provenientes de la parte alta de la vagina, cérvix, cuerpo uterino, tercio interno de las trompas, ligamento ancho, porción superior vesical, ciego, apéndice y parte terminal del intestino grueso, viajan por los simpáticos torácicos y lumbares, a través de los plexos cervicovaginales, uterino e hipogástrico hacia el nervio hipogástrico, a través del plexo hipogástrico superior, las cadenas simpática lumbar 
y torácica inferior; estos impulsos pasan luego por ramos blancos y comunicantes con relación a T11, T12 y L1 para seguir por las raíces dorsales de estos nervios para penetrar en la Médula a nivel de T11, T12 y L1 (3).

Figura 1

INERVACION TRACTO GENITAL FEMENINO

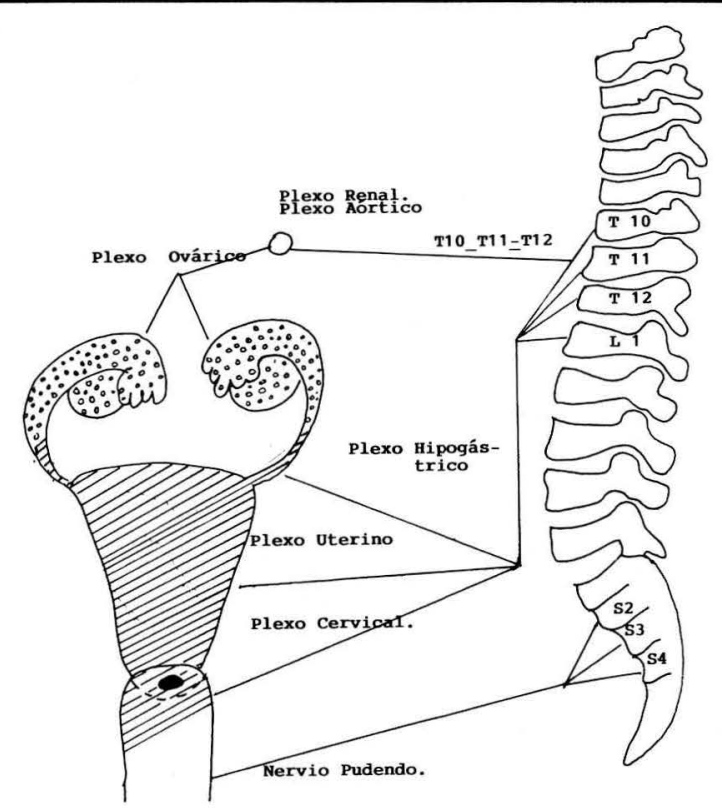

El plexo hipogástrico superior o nervio presacro no contiene aferentes provenientes del ovario ni de los tercios externos de la trompa, lo cual explica por que al hacer neurectomía presacra no hay alivio del dolor proveniente de los anexos.

\section{Características del dolor pélvico crónico ginecológico}

\section{Localización:}

El dolor crónico de origen ginecológico puede estar localizado en la región ventral o dorsal. La zona ventral se localiza en un lugar que no va más arriba del nivel de las espinas ilíacas anterosuperiores; la zona dorsal se localiza en la mitad superior del sacro extendiéndose lateralmente hacia los glúteos.

Figura 2

LOCALIZACION DOLOR PELVICO GINECOLOGICO

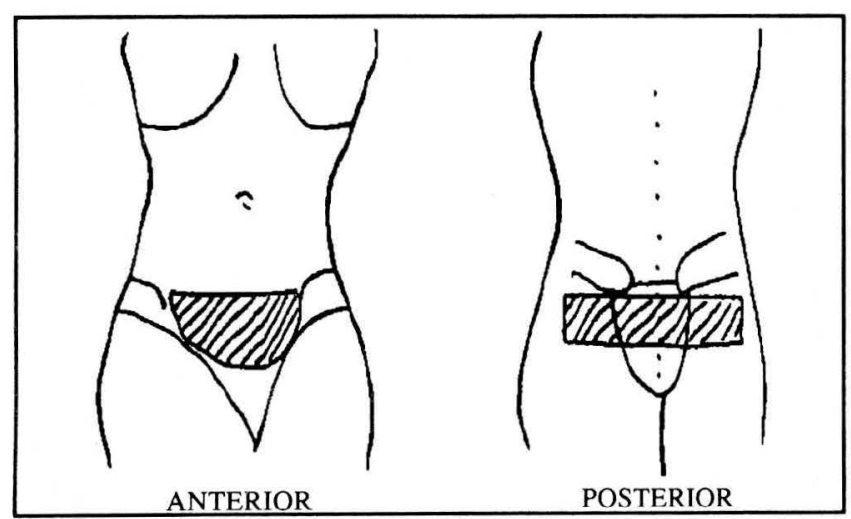

El dolor puede ser central (originado en útero, cérvix, ligamentos uterosacros y/o fondo de saco posterior), o puede ser lateral (originado en anexos) (3).

\section{Propagación}

El dolor de origen ginecológico especialmente el originado en el útero y menos frecuentemente originado en los anexos, tiende a propagarse a la parte anterior del muslo, muy rara vez a la parte interna o externa del mismo y nunca hacia la parte posterior. La propagación hacia la parte alta del abdomen casi nunca tiene origen ginecológico. La propagación a otros sitios puede ser debida indirectamente a entidades ginecológicas que por extenderse fuera de la pelvis (p.ej. tumor gigante de ovario) o que al comprometer otras estructuras (p.ej. cáncer ovárico con obstrucción intestinal), ocasionarán sintomatología de acuerdo con su localización y al órgano comprometido (3).

\section{Intensidad y relación con ciclo menstrual}

La intensidad del dolor es muy variable y puede tener fluctuaciones durante el ciclo menstrual, siendo en algunos casos típica su aparición al momento de la ovulación (Síndrome de Mittelschmerz); un dolor que se vuelve gradualmente más severo hacia el final de la menstruación en sugestivo de endometriosis; en algunos casos raros como el dolor ocasionado por parametritis o por retroversión uterina son más intensos en el período premenstrual y disminuyen al comienzo de la menstruación. Se debe recordar que ocasionalmente dolor de origen ortopédico muestra variaciones en los períodos premenstruales o menstruales (3).

Un síntoma muy frecuente al interrogar pacientes con DPC es la dispareunia superficial y/o profunda, relacionándose generalmente la segunda con alteraciones orgánicas.

\section{Enfoque diagnóstico}

El enfoque del DPC, se basa en una historia clínica muy detallada, enfatizando en las características, localización e irradiación del dolor.

En la Clínica de Dolor creada como grupo multidisciplinario en el IMI, se hace inicialmente una historia precodificada que contiene datos de identificación de la paciente , historia de la enfermedad actual, antecedentes, indagando específicamente sobre posibles factores psicógenos, trastornos del sueño, de la esfera sexual y demás áreas de importancia. Se realiza un examen físico completo, recordando la semiología del dolor de origen ginecológico y si es necesario se solicitarán paraclínicos.

Si el cuadro orienta hacia una dismenorrea primaria, no encontrándose patología orgánica, se inicia manejo médico, dejándose tiempo prudencial para evaluar mejoría y si no mejora, se pasará la paciente a endoscopia ginecológica (Cerivohisteroscopia-laparoscopia) en el mismo acto operatorio, haciendo una adecuada evaluación, buscando focos endometriósicos en zonas ocultas (fosas ováricas, repliegues peritoneales, bolsas peritoneales) y tomando biopsias de los sitios sospechosos de endometriosis no pigmentada.

Ante la sospecha de várices pélvicas y con endoscopia negativa se pasará a venografía (Ver Figs. 3 y 4). 
Figura 3.

DOLOR PELVICO CRONICO DIAGNOSTICO

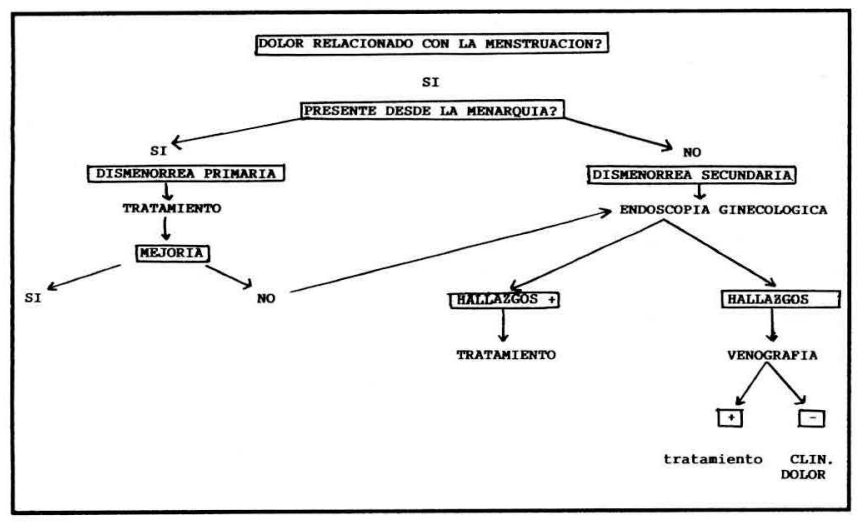

Figura 4.

DOLOR PELVICO CRONICO DIAGNOSTICO

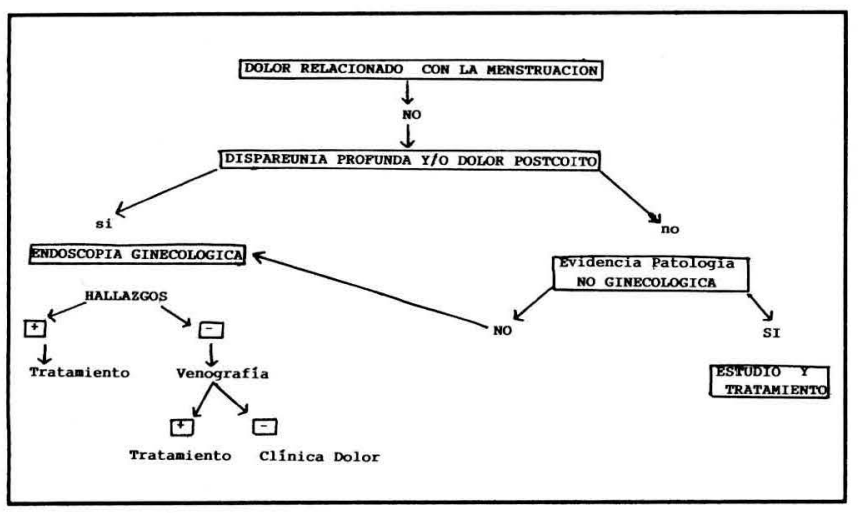

En toda paciente que consulte por DPC, así tenga una causa orgánica evidente, debe ser valorada por el grupo de Psicología Clínica, ya que la paciente como consecuencia de los trastornos producidos por el dolor crónico, como las alteraciones en el sueño, puede tener períodos de depresión y/o ansiedad que deben manejarse, pues el enfoque del grupo de dolor está orientado a reintegrar totalmente la paciente a sus actividades, que pueden haberse modificado. Toda paciente con etiología psíquica u orgánica es susceptible de verse favorecida por psicoterapia de grupo y/o individual, anotando que en la mayoría de pacientes con DPC generalmente la etiología es mixta, con predominio de factores orgánicos o psíquicos y rara vez aparecen aislados.

Un recurso importante es la evaluación por Trabajo Social, que nos permite saber cómo se desenvuelve la paciente en el entorno familiar y nos ayuda para el tratamiento no sólo de la paciente, sino a veces del grupo familiar.

En conclusión es indudable, que el enfoque y manejo de la paciente con DPC debe ser realizado por un equipo que esté dispuesto a estudiar el dolor crónico, a tener la sabiduría y paciencia para ayudar a este tipo de pacientes que ya han percibido el fracaso de recursos terapéuticos y no pocas veces se comportan agresivamente con los examinadores.
El pilar diagnóstico además de la buena historia clínica, es el recurso de la endoscopia ginecológica, que debe ser realizada por personal entrenado en reconocer la patología pélvica que puede ocasionar dolor.

\section{Etiología}

\section{A. Causas no ginecológicas}

Urológicas: Litiasis, cistitis, síndrome uretral, etc.

Gastrointestinales: Colitis ulcerativa, diverticulitis, síndrome colon irritable, apendicitis crónica, cáncer intestinal, hernias, ciego móvil, etc.

Musculoesqueléticas: Espondilolistesis, osteoporosis, tuberculosis ósea, hernia discal, malformaciones sacras, ileítis condensante, necrosis vertebral aséptica, trauma, imbalance muscular, etc.

\section{B. Causas ginecológicas}

Dolor
recurrente
cíclico $\quad\left[\begin{array}{l}\text { A. Dismenorrea } \\ \text { B. Secundaria: }\end{array} \quad \begin{array}{l}\begin{array}{l}\text { Endometriosis } \\ \text { Adenomiosis } \\ \text { Miomatosis } \\ \text { Dismenorrea } \\ \text { obstructiva } \\ \text { (congénita o adquirida) } \\ \text { DIU } \\ \text { Psicógena }\end{array} \\ \text { 2. Sínd. Mittelschmerz } \\ \text { 3. Sínd. Tensión premenstrual } \\ \text { 4. Enf. Pélvica inflamatoria } \\ \text { - Parametritis crónica } \\ \text { - Salpingooforitis crónica } \\ \text { - Salpingitis tuberculosa } \\ \text { 5. Desplazamiento uterino } \\ \text { - Prolapso genital } \\ \text { - Retroversión uterina } \\ \text { 6. Dolor ovárico } \\ \text { - Síndrome de ovario remanente } \\ \text { - Ooforitis escleroquística } \\ \text { - Ooforitis, periooforitis crónica } \\ \text { - Tumores ováricos } \\ \text { - Síndrome ovario residual } \\ \text { 7. Dolor parietal } \\ \text { 8. Varicocele pélvico. }\end{array}\right.$

\section{Dismenorrea}

La dismenorrea es un procedimiento ginecológico muy común, se estima que más del $50 \%$ de las mujeres que menstrúan, tienen dismenorrea, 1 a 3 días cada mes; en USA, se pierden cerca de 600 millones de horas laborales al año por este problema (3).

Es importante diferenciar entre la primaria y la secundaria; si el dolor está simétricamente localizado y no se evidencia patología orgánica hablaremos de una dismenorrea primaria. 


\section{Dismenorrea primaria}

En la mayoría de los casos comienza pocas horas o medio día antes del flujo menstrual, siendo su duración de 48 a 72 horas. Su comienzo data de la menarquia o poco tiempo después, rara vez después de un año.

El dolor es tipo cólico, localizado en hipogastrio, puede irradiarse hacia la zona sacroglútea, y/o parte anterior de muslos. Una localización unilateral es muy inusual. Durante las primeras horas el dolor es severo mientras el sangrado es escaso y a medida que aumenta el flujo menstrual va disminuyendo el dolor. Cuando el dolor es muy severo puede acompañarse de náuseas, vómito y a veces diarrea.

El examen físico no demuestra patología orgánica, pero hasta en 10-15\% habrá patología no reconocida al examen físico. Si no hay embarazo la severidad de la dismenorrea primaria tiende a disminuir después de un número variable de años.

\section{Fisiopatología dismenorrea primaria}

Una teoría postula que el dolor es por la presión intermitente del contenido uterino en el istmo ricamente inervado, $y$ el dolor persiste hasta que el istmo está suficientemente dilatado para permitir el paso de la sangre menstrual. Muchos autores han encontrado en estas pacientes un alto tono ítsmico, el cual se encuentra normalmente en la fase secretora del ciclo y se mantiene hasta el comienzo de la menstruación, mientras que en mujeres sin dismenorrea el tono ítsmico disminuye 24 horas o más antes de la menstruación.

Se ha encontrado elevada concentración de prostaglandinas en endometrio y sangre menstrual de pacientes con dismenorrea primaria y es probable que el dolor se deba a persistencia de la hipertonía del istmo con un impedimento temporal funcional al flujo menstrual, lo cual combinado con aumento de la producción de prostaglandinas lleva a un incremento de contracciones miometriales disrrítmicas, sensibilización de nervios terminales a prostaglandinas e isquemia de la pared uterina; sin embargo no se pueden descartar en su etiopatogenia los factores psicógenos.

\section{Dismenorrea secundaria}

Se denomina dismenorrea secundaria cuando hay causa orgánica responsable del dolor. Muchas veces el dolor es unilateral, y el comienzo es después de la menarquia.

a. Endometriosis: Es la presencia de focos endometriales ectópicos, ya sea en fondo de saco de Douglas, en ovarios o en superficie posterior de ligamento ancho y más raramente en sitios como sigmoide, intestino delgado, etc.

Estos focos pueden o no ser pigmentados.

Cerca del $40 \%$ de los focos ectópicos permanecen sin sintomatología, pero en los casos manifiestos el principal síntoma es el dolor en forma de dismenorrea o síndrome de tensión premenstrual, con exacerbación durante la fase menstrual y dolor continuo con o sin exacerbación menstrual.

b. Adenomiosis: Es la presencia de endometrio en el miometrio.

Se encuentra en el 3-8\% de los especímenes de histerectomías; el 50\% de las pacientes están en la 5a. década (3).
La sintomatología más común es la presencia de monometrorragias y dismenorrea, pero ambos síntomas coinciden en solamente el $20 \%$ (3).

c. Miomatosis: Los miomas son una patología muy frecuente después de los 30 años, rara vez producen dismenorrea pero cuando lo hacen es por su localización submucosa.

d. Dismenorrea osbtructiva: Puede ser:

Congénita: Por atresia de himen, septum transverso vaginal, atresia cervical, cuerno rudimentario, útero doble no comunicado, etc.

Adquirida: Por adherencias intracervicales o adherencias intrauterinas.

\section{Síndrome Mittelschmerz}

Es el dolor al momento de la ovulación y es el motivo de consulta del 1-3\% de las pacientes que asisten a la consulta ginecológica, siendo más frecuente en edades entre 20-30 años. El dolor puede ser en una fosa ilíaca o en ambas y también alternadamente.

\section{Síndrome de tensión premenstrual}

Es el nombre de un síndrome vago de un variable número de síntomas que aparecen en forma cíclica 8-14 días antes de la menstruación y desaparece al inicio de ésta.

Síntomas principales son una sensación de tensión de los senos y del abdomen; muchas presentan tensión nerviosa, inestabilidad emocional, ansiedad, depresión e insomnio. Es más frecuente alrededor de los 30 años.

\section{Enfermedad pélvica inflamatoria}

a. Parametritis crónica: La cervicitis crónica no es dolorosa pero en algunas instancias provoca dolor pélvico espontáneo y más frecuentemente dispareunia profunda.

Se puede sospechar cuando hay dispareunia en las pacientes con cervicitis crónica, dolor a la movilización cervical y prácticamente se hace una prueba terapéutica con antibióticos y antiinflamatorios.

b. Salpingooforitis crónica.

c. Salpingitis tuberculosa.

\section{Desplazamiento uterino}

a. Prolapso genital: Usualmente no produce dolor pero por efecto de la tracción de las diversas estructuras puede producir molestias en el abdomen bajo, debido a trastornos circulatorios por la malposición.

b. Retroversión fija: Generalmente es secundaria a entidades que producen adherencias, como la endometriosis o secuelas de enfermedad pélvica inflamatoria.

c. Retroversión móvil: Usualmente no causa sintomatología, pero algunos casos pueden llevar a dolor intermitente con o sin dispareunia. El dolor intermenstrual puede localizarse en el abdomen bajo y/o en región sacroglútea; el dolor generalmente comienza después de un parto o un aborto. El dolor empeora en el período premenstrual y disminuye o desaparece después del primer o segundo día menstrual e igualmente con el reposo. 


\section{Dolor ovárico}

a. Síndrome de ovario remanente: Puede ser visto en pacientes a quienes se les ha hecho ooforectomía bilateral, pero por dificultades casi siempre técnicas han quedado residuos de ovario como en el caso de extensas lesiones endometriósicas con amplias adherencias. La laparoscopia suele ser no muy útil y sólo se sospecha cuando la paciente tiene evidencia de secreción estrogénica que persiste después de dar corticoides, para suprimir la secreción de androstendiona adrenal y su conversión periférica a estrógenos. El tratamiento suele ser una excisión meticulosa del tejido ovárico residual.

b. Ooforitis escleroquística: Este es un síndrome descrito por autores franceses que describe con dolor en fosa ilíaca uni o bilateral, ciclos menstruales irregulares y uno o dos ovarios agrandados. Hoy ha sido denominada esta entidad distrofia ovárica dolorosa.

\section{c. Ooforitis, periooforitis crónica}

d. Tumores ováricos: Generalmente los tumores ováricos benignos no son dolorosos a menos que sufran alguna complicación como torsión, hemorragia intraquística; e igual sucede con los tumores malignos aunque éstos últimos pueden producir dolor indirectamente cuando el crecimiento extracapsular interfiere con la función de órganos adyacentes.

e. Síndrome de ovario residual: En casos de histerectomías donde se dejan in-situ los ovarios puede haber DPC, conformando el síndrome de ovario residual cuando se han descartado otras causas; frecuentemente se encuentran al explorar adherencias pélvicas y periooforitis.

\section{Dolor parietal}

Puede ser debido a varias causas que irriten los nervios espinales de los segmentos T7-L1.

El estímulo que provoca dolor puede estar localizado en las columnas posteriores de la médula espinal, las raíces posteriores y ganglios espinales o nervios periféricos.

El dolor parietal puede ser debido a:

a. Cicatrices quirúrgicas: Especialmente transversas como apendicectomías, nefrectomías, herniotomía o incisiones de Pfannenstiel (al atrapar nervios ileohipogástricos).

b. Endometriosis: Localizada en la pared abdominal.

c. Hematoma de los rectos: especialmente en pacientes viejos o que reciben corticoides por largo tiempo.

d. Neuropatía por atrapamiento: Ocasionada porque en el transcurso de los nervios ileohipogástrico, quedan «atrapados», en el sitio donde perforan la fascia y músculos de la pared abdominal. Esto es raro pero puede verse en las últimas semanas del embarazo.

\section{Várices pélvicas}

El dolor sin patología obvia es un problema no poco frecuente en Ginecología. En Inglaterra de 20971 laparoscopias, 10823 fueron por dolor pélvico, pero debemos anotar que en el $63 \%$ de éstas no se encontró patología demostrable (4); es por esto, que en el algoritmo diagnóstico de nuestra clínica de dolor cuando la endoscopia es negativa, sugerimos estudio venográfico, para descartar várices pélvicas cuya presencia es importante cuando la endoscopia ha sido negativa, como lo demuestran los estudios británicos al respecto presentados en el XXIV Congreso Británico de Ginecología y Obstetricia realizado en 1986.

Esta patología es más frecuente en pacientes multíparas, ocasionando dolor pélvico, que aumenta con la bipedestación y que mejora con el reposo; al examen físico el dolor se puede reproducir al comprimir los puntos ováricos, localizados en la unión de $1 / 3$ superior con $2 / 3$ inferiores de una línea imaginaria que una al ombligo con las espinas ilíacas anterosuperiores (ver figura No. 5), quizá por comprimir la vena ovárica (4).

Figura 5

LOCALIZACION DE PUNTOS OVARICOS

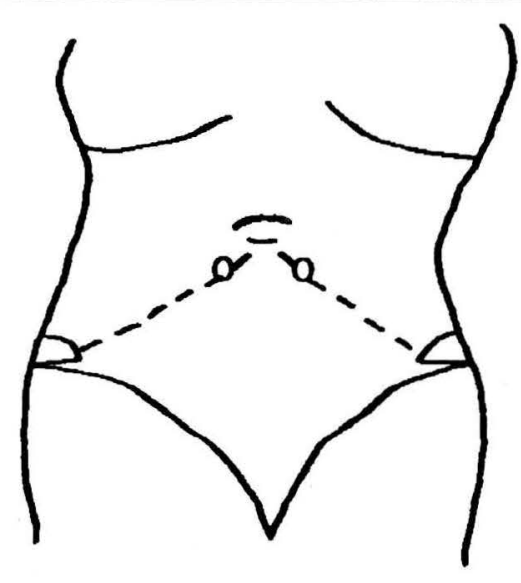

0: PUNTOS OVARICOS

La histología de estas venas es similar a la de las várices de miembros inferiores, encontrando fibrosis de la túnica media e íntima, hipertrofia de la muscular y proliferación del endotelio capilar.

Podemos especular 3 posibles etiologías:

1. Mecanismo similar a las várices de miembros inferiores, como defecto valvular, multiparidad, etc.

2. La segunda posibilidad es que un incremento anormal del flujo en una región con muchas conexiones arteriovenosas, como la pelvis, puede llevar a un estado de dilatación venosa crónica pélvica. Algunos autores sugieren que la congestión pélvica es un trastorno del sistema nervioso autónomo pélvico en pacientes predispuestas psiquiátricamente.

Así siendo la pelvis un sitio de rápidos cambios en el flujo sanguíneo, teniendo un mal soporte venoso y con una base de stress, pueden originarse varicosidades.

Muchas de estas pacientes tienen historia de stress desde la infancia.

3. La tercera posibilidad, es que resulten de la relajación del músculo liso en las paredes de las venas pélvicas, causada por alguna sustancia vasoactiva aún no identificada. Estos tres mecanismos pueden actuar individualmente o juntos.

La historia clínica orienta, al igual que el reproducir el dolor, al comprimir los puntos ováricos; ocasionalmente en la laparoscopia se pueden ver las dilataciones varicosas, en la ecografía se puede igualmente sospechar, pero el diagnóstico menos incierto se hace por venografía. 
Hay por lo menos 6 técnicas diferentes de venografía pélvica pero la menos molesta y más efectiva es la técnica transuterina sin compresión abdominal. Se da un puntaje en la venografía de 1-3, a tres parámetros: diámetro máximo de la vena ovárica, tiempo de desaparición del medio de contraste y el grado de congestión del plejo ovárico. Un puntaje de 5 o más es diagnóstico. La sensibilidad de esta prueba es del $91 \%$ y tiene una especificidad del $89 \%$ (4).

Aún no hay un tratamiento bien establecido, algunos postulan un tratamiento quirúrgico que varía desde excisión de las várices visibles hasta histerectomía.
Hay descritos tratamientos farmacológicos con progestágenos o deshidroergotamina, ésta última por efecto en el lado arterial del árbol vascular; aunque también es de anotar que antagoniza la 5 hidroxitriptamina lo cual puede explicar en parte su acción analgésica, además es agonista de receptores adrenérgicos (5). Como ya se expuso esta entidad tiene amplias bases psicosomáticas, por lo cual la psicoterapia puede ser gran coadyuvante terapéutico.

\section{BIBLIOGRAFIA}

1. Dicker RC., Greenspan JR., Strauss LT et al. Confirmation of the preoperative diagnosis for Hysterectomy. Am. J. Obstet. Gynecol. 1984; 150: 283.

2. Gómez PI., Riaño JE. Boletín Clínica de Dolor Pélvico Crónico. Instituto Materno Infantil de Bogotá. 1990; 1(1).

3. Gómez PI. Dolor Pélvico Crónico en Ginecología. Boletín Clínica de Dolor Pélvico Crónico. Instituto Materno Infantil de Bogotá, 1990; 1(2).
4. Beard R., Highman J., Pearce S., Reginald P. Diagnosis of Pelvic Varicosities in women with Chronic Pelvic Pain. The Lancet 1984; 946-949.

5. Barton D. Dihydroergotamine and the pelvic pain syndrome. Letter to Editor. The Lancet 1987; 857. 